\title{
Proteomic Study of Hepatic Nuclear Extracts in an Adaptive Acetaminophen Tolerance Model
}

\author{
Pamela S. Steele • Mark W. Linder • Roland Valdes Jr. • \\ Deanna D. H. Franke • Saeed A. Jortani
}

Published online: 3 February 2009

(C) Humana Press 2009

\begin{abstract}
Introduction Variability in response to acetaminophen (APAP)-induced aseptic inflammation and tolerance to the impending hepatic damage has been described. To understand the mechanism of adaptive tolerance, we investigated the proteomic profiles of crude nuclear lysates in a mouse model. We hypothesized that pretreatment with low doses of APAP prior to a toxic dose results in differential protein expression.

Materials and Methods Mice (BALB/C) were separated into three groups: the pretreated (PT) group received incremental doses of APAP while the last dose only (LD) and naïve groups were given saline vehicle. A toxic dose of APAP was administered on the seventh day to the PT and LD animals only and all groups were euthanized $3 \mathrm{~h}$ postdose. Total protein from crude hepatic nuclear lysates were applied to protein arrays and analyzed by immunoaffinity mass spectrometry.

Results and Discussion Comparative data analyses of protein peaks revealed a protein that was significantly increased at $\mathrm{m} / \mathrm{z}$ of 60,030 (p60) in the LD animals vs the other two groups. The closest match for the preliminary identification of the p60 protein based on a Swiss-Prot/ TagIdent database search using the approximate isoelectric point and molecular weight information was Ccr4-Not complex subunit-2. This protein is a subunit of a multiprotein complex and serves as a transcriptional suppressor involved in controlling mRNA synthesis and degradation.
\end{abstract}

P. S. Steele $\cdot$ M. W. Linder $\cdot$ R. Valdes Jr. D. D. H. Franke

S. A. Jortani $(\square)$

Department of Pathology and Laboratory Medicine,

School of Medicine, University of Louisville,

Louisville, KY 40202, USA

e-mail: sjortani@louisville.edu
Preliminary identification was also supported by Western blot analysis using anti-CNOT2 antibody.

Conclusion Considering the APAP tolerance model, we conclude that toxicogenomic approaches such as nuclear profiling are useful tools in assessing differential expression of transcriptional factors involved in inflammatory response and adaptive tolerance to toxins.

Keywords Aseptic inflammation - Liver Proteomic . Adaptive tolerance - Acetaminophen - Toxicogenomic . Ccr4-Not subunit-2 Transcriptional factor $\cdot$ Mouse $\cdot$ Nuclear

\section{Introduction}

Acetaminophen ( $N$-acetyl-para-aminophenol or APAP) is a commonly available over-the-counter medication. At therapeutic doses, it is an effective analgesic and antipyretic; however, when consumed in large quantities due to accidental or deliberate overdose, APAP is the leading cause of drug-induced acute liver failure [1]. As reviewed by Gelotte et al. [2], approximately $90 \%$ of APAP is metabolized by two rate-limiting factors. The metabolism of both glucuronidation via uridine 5'-diphospho-glucuronosyltransferase (isoform UGT1A6) and sulfotransferase isoforms (SULT1A1 and 1A3) are based upon the availability of their respective inorganic forms and enzymatic transferase activities. Roughly $8-10 \%$ of APAP is metabolized by cytochrome P450 (CYP), a superfamily of hemoproteins involved in the metabolism of steroid hormones, drugs, and xenobiotic substances, and the remaining fraction is excreted unchanged by the kidneys. The primary cytochrome involved in APAP metabolism, CYP2E1, results in the formation of a highly reactive and toxic intermediate $N$-acetyl- $p$-benzo-quinone imine 
(NAPQI) that is subsequently metabolized to glutathione by glutathione- $S$-transferases. Thiol metabolites of glutathione production include cysteine, mercapturate, methylthioacetaminophen, and methyanesulfinylacetaminophen [2] which are excreted thru the bile or urinary system. In conditions of APAP overdose and oxidative stress, the primary metabolic pathway becomes saturated. The reduced glutathione stores are rapidly depleted and thereby NAPQI accumulates at the site of metabolism, the liver. As a reactive intermediate, NAPQI is then free to covalently bind to various intracellular targets, ultimately resulting in hepatocellular injury and necrosis.

Although the exact mechanism by which APAP exerts toxicity is not known, it is clear that liver susceptibility to APAP toxicity is multifactorial. Furthermore, it is yet to be determined how some individuals tolerate extreme doses of APAP without exhibiting hepatic toxicity. One such case was reported by Shayiq and colleagues [3] involving a patient with an estimated daily ingestion of $65 \mathrm{~g}$ of APAP. While this massive ingestion was thought to have exhibited extensive hepatic necrosis, permanent liver injury did not occur. This lack of toxicity may be attributed in part to inherent variations in CYP2E1 expression [4, 5]. Animal models have been useful in the studies of complex cellular responses and networks involved in toxicity. Using these approaches, induction of tolerance to poisoning brought about by incremental exposure to multiple sublethal doses (prior to a lethal dose) have been investigated. To elucidate the hepatoprotective nature of this exposure, Shayiq et al. [3] developed a mouse model in which animals were either pretreated with saline vehicle or administered incremental sublethal doses of APAP prior to the administration of a lethal dose. Induced tolerance to APAP was measured in terms of the median lethal dose (LD-50) or the dose of a toxic agent necessary to kill $50 \%$ of the study population in a defined time interval. In this study, the LD-50 of the APAP pretreatment group increased by $400 \%$, suggesting that pre-exposure afforded hepatoprotection. We have reproduced this model of adaptive tolerance in our laboratory and have confirmed tolerance of the pretreated animals by demonstrating a lack in increased serum alanine transaminase (ALT) concentrations in the pretreated animals under APAP dosing conditions that lead to fivefold increased serum ALT in naïve animals [6].

Proposals for hepatocyte autoprotection (i.e., induced or adaptive tolerance) include the role of oxidative stress [79], inflammatory chemokines and cytokines [10-12], and the resultant equilibrium shift in the balance between proinflammatory and anti-inflammatory cytokines [8, 9]. The investigation of covalent bonding of APAP intermediates to enzymes and proteins has lead to the examination of glutathione expression [13] and hepatocyte regeneration $[10,11,14]$.
A variety of standard techniques have been utilized to assess potential molecular mechanisms of adaptive tolerance, including immunohistochemistry [8, 9], RNA, and protein analyses [14, 15]. Recent technological advancements in proteomic profiling and bioinformatics have allowed for a dramatic increase in the analyses of biomarkers in complex biological fluids including: serum and plasma [16-19], urine [20-23], cellular subfractions [24-26], ligand binding [27-29], and cerebral spinal fluid [30, 31]. Although these applications have systematic limitations [32-34], a variety of platforms are being used for the separation and identification of novel proteins involved in various cellular processes.

In an effort to understand and characterize the mechanism of adaptive tolerance, we have utilized proteomic analyses to evaluate protein expression in crude nuclear lysates by bioaffinity mass spectrometry. Using a mouse model of APAP toxicity established in our laboratory [28], we hypothesized that animals pretreated with low doses of this drug prior to a final toxic dose differentially express proteins compared to naïve animals and, as such, these proteins may contribute to the observed hepatoprotection in our model system. Understanding the mechanisms for adaptive tolerance to drugs may provide insights into better diagnoses and treatments for drug toxicities.

\section{Materials and Methods}

\section{Animal Regimen}

Animal studies were approved by the University of Louisville Institutional Animal Care and Use Committee (IACUC). Eighteen male BALB/C mice (The Jackson Laboratory, Bar Harbor, ME, USA) were randomly divided into three groups (Fig. 1). Briefly, the last dose only group (LD) received $0.9 \%$ intraperitoneal (i.p.) saline injections for 6 days and received the lethal dose $(350 \mathrm{mg} / \mathrm{kg})$ on the seventh day. The pretreated group (PT) received incremental doses of APAP followed by administration of a lethal dose on the seventh day. The control group (naïve) received i.p. saline vehicle only for the duration of the study. Animals were euthanized by cervical dislocation $3 \mathrm{~h}$ postinjection and their liver tissues were immediately perfused with $5 \mathrm{~mL}$ of ice-cold saline, excised, and minced with scissors in a beaker of ice-cold saline.

\section{Sample Preparation}

The saline solution was drained from minced tissue and the remaining tissue fragments were homogenized in $0.25 \mathrm{M}$ sucrose buffered with $50 \mathrm{mM}$ potassium phosphate buffer ( $\mathrm{pH}$ 7.4). Homogenates were centrifuged at $1,000 \mathrm{rpm}$ in

\section{藻 Humana Press}




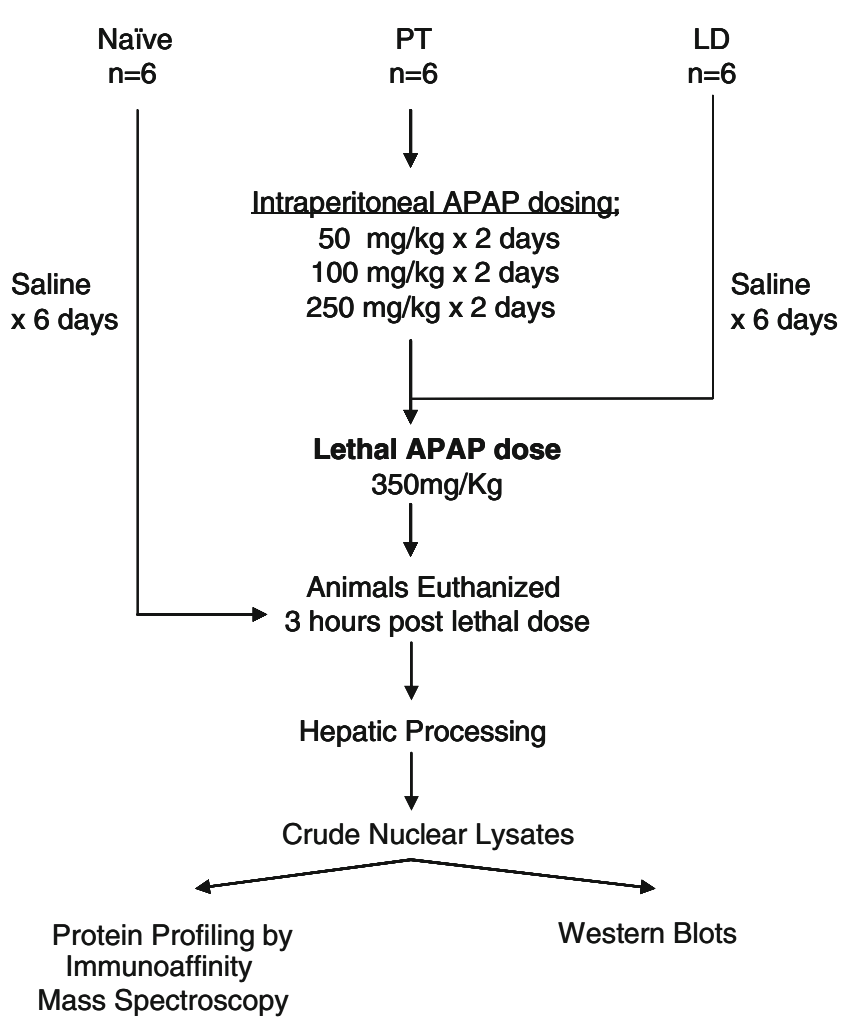

Fig. 1 APAP dosing regimen. The naïve group was treated with saline vehicle only. The pretreated group $(P T)$ was incrementally administered various doses of APAP followed by a lethal dose. The last dose only animals $(L D)$ were treated with saline followed by a final lethal dose of APAP

$50 \mathrm{~mL}$ polycarbonate tubes to sediment cellular debris. The supernatant was then resedimented at $12,000 \mathrm{rpm}$ for $20 \mathrm{~min}$. Crude nuclear pellets were then aliquoted and stored frozen at $-80^{\circ} \mathrm{C}$.

Frozen pellets (approximately $700 \mu \mathrm{L}$ ) were thawed on ice and combined with approximately $300 \mu \mathrm{L}$ of autoclaved, double-distilled ice-cold $\mathrm{H}_{2} \mathrm{O}$ containing protease inhibitors (Complete Protease Inhibitors; Roche \#1836153; 1:7 dilution, tablet to $\mathrm{H}_{2} \mathrm{O}$ ). The resultant volume was vortexed briefly and the nuclear suspension (approximately $200 \mu \mathrm{L}$ ) was removed and transferred to $300 \mu \mathrm{L}$ of $\mathrm{U} 9$ buffer (9 M urea, 2\% 3-[(3-cholamidopropyl) dimethylammonio]propanesulfonic acid, $50 \mathrm{mM}$ Tris- $\mathrm{HCl}, \mathrm{pH}$ 9.0). Samples were then vortexed approximately $10 \mathrm{~min}$ at room temperature and then centrifuged at $20,000 \times g$ at $4^{\circ} \mathrm{C}$ for $30 \mathrm{~min}$. The supernatants were collected, aliquoted on ice, and immediately stored at $-80^{\circ} \mathrm{C}[35]$.

\section{Chip Preparation}

Immunoaffinity mass spectrometry products were obtained from Bio-Rad Laboratories (Hercules, CA, USA) including: chip arrays, bioprocessors (with disposable reservoirs and gaskets), sinapinic acid (50\% acetonitrile, $0.5 \%$ trifluoro- acetic acid [SPA]) as the energy absorbing molecules (EAM), PBS-II reader (time of flight mass spectrometer [TOF-MS]), and the Biomarker Wizard (BW) software. The performance of the mass spectrometer was monitored weekly by assessing its calibration according to the manufacturer's instructions.

Multiple chip arrays were utilized to capture proteins based upon their innate biochemical properties, including IMAC30 (metal binding), SAX2 (strong cation exchange), and $\mathrm{H} 4$ (hydrophobic) surfaces. Chips were processed in 96-well formatted bioprocessors. The IMAC30 chip surface required activation by the addition of $100 \mathrm{mM} \mathrm{CuSO}_{4}$; the addition of $100 \mathrm{mM}$ of sodium bicarbonate $(\mathrm{pH} 4.0)$ facilitated the removal of any remaining unbound copper molecules. All chip types were equilibrated with their respective binding/washing buffers according to the manufacturer's instructions. Binding/washing buffers for IMAC30 (100 mM NaH ${ }_{2} \mathrm{PO}_{4}$ [pH 7.0], $0.5 \mathrm{M} \mathrm{NaCl}, 0.1 \%$ polyethylene glycol $p$-(1,1,3,3-tetramethylbutyl)-phenyl ether [Triton X-100]), SAX2 (10 mM Tris Base [pH 8.0], $0.1 \%$ Triton $\mathrm{X}-100)$ and $\mathrm{H} 4(50 \mathrm{mM} 1 \mathrm{mM} \mathrm{N}-(2-$ hydroxyethyl)-piperazine- $N{ }^{\prime}$-2-ethanesulfonic acid [HEPES buffer, $\mathrm{pH}$ 7.0], 0.1\% Triton X-100) surfaces were applied to the chip surface prior to protein application.

Protein concentration was determined using the Bradford Assay (Bio-Rad Laboratories, Hercules, CA, USA) using albumin as the standard (Sigma-Aldrich [purity of $96 \%$ by electrophoresis]). Crude nuclear lysates $(15 \mu \mathrm{g}$ in 2:3 $[v / v]$ of deionized water/U9 buffer) were incubated on chip surfaces for 30 min followed by repeated washes with each chip's respective buffer. All chips were washed with $1 \mathrm{mM}$ HEPES buffer ( $\mathrm{pH}$ 7.0) prior to bioprocessor disassembly. Excess volume was drained from each chip spot and arrays were air-dried. The EAM for each chip type was SPA and was applied in two successive $1-\mu \mathrm{L}$ aliquots. Experimental groups were analyzed concurrently to minimize chip to chip variation using the Bio-Rad Laboratories PBS-II protein chip reader. Samples were randomized on each chip and run in triplicate.

Initially, all spectra were visually inspected. If the matrix peaks were missing or depressed below $30 \%$ the lognormalized value, the spectra were eliminated from final data analysis. Peaks were detected with first and second pass signal to noise ratio of 5 and 2 using the BW software. All spectra were normalized according to manufacturer's instructions. Calculated $p$ values using the BW software were utilized in selecting proteins which yielded the greatest mass difference amongst groups. The mass of relevant peaks were further analyzed using the ExPASy molecular biology server (http://ca.expasy.org/tools/tagi dent.html; accessed on March 2005) to ascertain potential protein candidates. Input variables included a percentage range about the molecular weight (MW), isoelectric point 
(pI), range of the protein (as estimated by the $\mathrm{pH}$ of the binding and wash buffer solutions for each chip type), organism classification (i.e., mouse), and keyword (restricted based on UniProtKB list of keywords).

\section{Western Blot}

Mouse liver crude nuclear lysates $(15 \mu \mathrm{g}$ per lane) were loaded onto $4-20 \%$ gels (Bio-Rad Laboratories, Hercules, CA, USA) prior to sodium dodecyl sulfate polyacrylamide gel electrophoresis. Gels were transferred to nitrocellulose membrane using the Semi-Dry Trans-Blot Apparatus (BioRad Laboratories, Hercules, CA, USA) according to manufacturer's instructions. Following an initial block (5\% dried milk in $1 \mathrm{X}$ phosphate buffered saline, $137 \mathrm{mM}$ $\mathrm{NaCl}, 10 \mathrm{mM}$ phosphate, $2.7 \mathrm{mM} \mathrm{KCl}, \mathrm{pH} 7.4)$ and multiple washes (1X phosphate buffered saline plus $0.005 \%$ Tween-20), membranes were hybridized with a rabbit antihuman IgG polyclonal antibody to CNOT2 using a 1:500 dilution in blocking buffer (affinity purified recombinant protein, confirmed by immunohistochemistry and Western blotting [ProteinTech Group, Chicago, IL, USA]) [35]. Membranes were then washed with agitation for $30 \mathrm{~min}$ and subsequently hybridized with a goat antirabbit IgG-labeled HRP antibody diluted 1:2,000 in blocking buffer (Abcam, Cambridge, MA, USA). Chemiluminescent signal was detected by Enhanced Chemiluminescent Plus reagents according to the manufacturer's recommendations (Amersham Biosciences, Piscataway, NJ, USA) and exposed to chemiluminescence film.

\section{Results and Discussion}

Crude hepatic nuclear lysates from the three experimental conditions (i.e., naïve, PT, LD) were analyzed on IMAC30, $\mathrm{H} 4$, and SAX2 chip types. The ranked $p$ values for the detected peaks (as calculated by the BW software) for all chip types were analyzed in order to select proteins that were significantly altered with the most probable differ-

Table 1 Analyses of chip types vs treatment groups

\begin{tabular}{lccr}
\hline Group & \multicolumn{2}{l}{ Chip type } & \\
\cline { 2 - 4 } & SAX2 & IMAQ & H4 \\
\hline LD vs PT & 23 & 10 & 20 \\
Naïve vs PT & 7 & 5 & 19 \\
LD vs Naïve & 6 & 2 & 7
\end{tabular}

Chip types included: IMAC30 (metal binding), SAX2 (strong cation exchange), and H4 (hydrophobic) surfaces. Treatment groups included: naïve (saline vehicle only), LD (saline vehicle/last dose only), and PT (pretreatment with incremental doses of APAP followed by a lethal dose). All $p$ values $\leq 0.05$

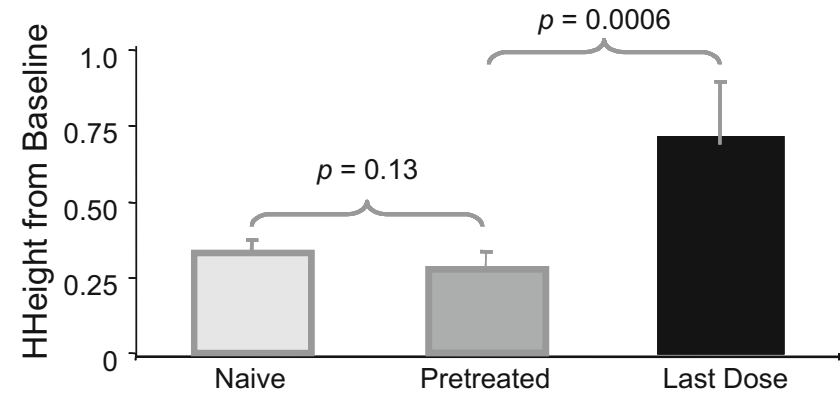

Fig. 2 Comparison of peaks (height from baseline) for the three groups. The height from baseline of the p60 protein was significantly different between the PT and LD groups $(p=0.0006)$ but not between the naive and PT animals $(p=0.13)$. Standard deviations are shown

ences amongst the groups. For example, proteins scanned between $\mathrm{m} / \mathrm{z} 1,643$ and 125,128 on the SAX2 chip yielded 65 protein peaks. Since it would have been exhaustive to identify all peaks from the three chips with signal to noise ratios $>3$, we only pursued peaks with $p$ values $\leq 0.05$ for comparison among experimental groups (Table 1). Peaks that were significantly different were further queried using the Swiss-Prot database for potentially relevant nuclear protein candidates (transcription factors) involved in adaptive tolerance.

Our investigations lead us to focus primarily on the SAX2 chip protein peaks. We chose to concentrate on the preliminary identification steps for one particular protein with a $\mathrm{m} / \mathrm{z}$ of $60,030 \mathrm{Da}(\mathrm{p} 60)$. The p60 protein was significantly increased in the LD (Fig. 2), which exhibited the greatest toxicity after the final dose of $350 \mathrm{mg} / \mathrm{kg}$ APAP ( $p$ value $=0.0006)$. The $\mathrm{p} 60$ protein in animals receiving the LD was also significantly increased ( $p$ value $=0.0032$ ) when compared to the naïve group. Interestingly, this protein was not significantly different between the naïve and the PT group ( $p$ value $=0.1278$ ).

Tentative protein identification via the ExPASy SwissProt/TagIdent database allowed for the incorporation of $\mathrm{pI}$, MW, organism classification, and a set of predefined key words. Since this protein had been discovered using the SAX2 chip type with a binding/washing buffer with a $\mathrm{pH}$

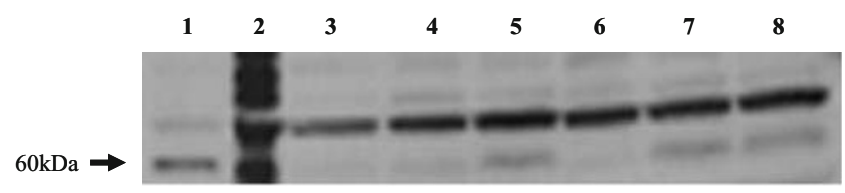

Fig. 3 The p60 protein analyses by Western blot. We verified the putative match by using antibody specific to the Ccr4-Not transcription complex subunit-2 protein. Lane 1 the positive control ( $\mathrm{HeLa}$ cells), lane 2 the MW standards. Independent samples are depicted for naïve (lanes 3-4), pretreated (lanes 5-6), and last dose only (lanes 7 8 ) animals. The negative control did not contain any bands and is not shown. These results indicate that the CNOT2 protein is differentially expressed, consistent with mass spectrometry data 
of 8.0, the following specifications were used as search criteria: MW of $60.030 \pm 600$, pI equal to $8.0 \pm 1.0$, and organism classification defined as mouse. The ExPASy Swiss-Prot database resulted in 39 possible matches. Elimination criteria included proteins not expressed in the nucleus and from tissues other than the liver. The carbon catabolite repressor 4-negative on TATA-less genes (Ccr4Not transcription complex) subunit-2 was determined to be the best fit and closest partial identification for this protein based on the pI (7.61), MW (59,711), and organism classification (mouse).

The NOT genes were originally isolated in yeast $[36,37]$ by genetic screening and identified as negative regulatory elements of transcription on TATA-less promoters (HIS3 gene; $S$. cerevisiae). The $C N O T 2$ gene, a member of the CNOT 2/3/5 family, is located on chromosome 10:115889276-115985621 bp, - strand (GeneID: 72068) in mice (Cnot2) and on 12q15 (GeneID: 4848) in humans (CNOT2). There are two forms of the Ccr4-Not complex (1.0 and 1.9 MDa forms) as determined by mass spectrometry [38]. As reviewed by Denis and Chen [39], the roles of the Ccr4-Not transcriptional complex include the repression and activation of mRNA initiation, mRNA elongation, deadenylation, and degradation. This complex signaling network can function individually or synergistically to initiate various functions [39] and is located in both in the nucleus and cytoplasm [40]. Polymorphisms are known to influence transcriptional activity; human CNOT2 has approximately 300 single-nucleotide polymorphisms/variants as identified by Applied Biosystems (Foster City, CA, USA; http://www.genecards.org/cgi-bin/carddisp.pl?gene= CNOT2), while the mouse (Mus musculus) has eight (http://www.ncbi.nlm.nih.gov/SNP/snp_ref.cgi?locusId= 72068).

To further test the validity of the preliminary identity of the p60 protein being CNOT2, we purchased a commercially available anti-CNOT2 polyclonal antibody. Preliminary Western blot results from independent animal samples from naïve, PT, and LD mice (Fig. 3) resulted in crossreactivity of multiple bands. As the Ccr4-Not complex contains many proteins that span a defined MW region, we anticipated cross-reactivity consistent with those previously reported by Winkler et al. [40]. However, the p60 protein illustrated a trend toward an increased expression in the LD group compared to the naïve and PT mice. These results are consistent with the mass spectrometric findings (above) in that the naïve and PT protein levels did not significantly differ from each other but their protein expression was lower than the LD group (Fig. 2). Together, these findings are consistent with the lack of APAP toxicity in PT animals as measured by serum ALT concentrations [6]. It appears that the PT animals developed an adaptive tolerance to APAP due to the incremental dosing over the experimental period. Interestingly, without pretreatment, the LD group exhibited widespread hepatic inflammation and necrosis [41].

Our preliminary identification of this protein as CNOT2 suggests that its expression may ultimately result in regulatory factors that mediate APAP-induced hepatic inflammation. Inflammation of major organs such as the liver and kidneys is the primary means by which APAP exerts its initial toxic effects $[6,42,43]$. In APAP poisoning cases, it takes 2-3 days on average for hepatic inflammation and damage to appear clinically. The finding that the CNOT2 protein was expressed similarly in the naive and PT animals implies a potential role for this transcriptional factor in adaptive APAP tolerance. It is clear from the animal model that proinflammatory cytokines play a major role in APAP adaptive tolerance [11, 44-47]. Identification of the p60 protein via proteomic technology has augmented our knowledge of the potential key players in aseptic inflammation. The involvement of a transcriptional factor regulating gene expression in adaptive APAP tolerance is suggestive of transcriptional control as a regulatory mechanism of autoprotection.

Acknowledgements The authors would like to thank Dr. Christine Simmons, Mr. Matt Herrnberger, and Dr. Marjorie Bon Homme for their excellent technical assistance. Support for these studies was provided by the following grants: NIH 1P30ES014443-01A1 and NIH 1-RO1-GM065459.

\section{References}

1. Lee WM. Acetaminophen and the U.S. Acute Liver Failure Study Group: lowering the risks of hepatic failure. Hepatology (Baltimore, Md.) 2004;40(1):6-9.

2. Gelotte CK, Auiler JF, Lynch JM, et al. Disposition of acetaminophen at 4,6 , and $8 \mathrm{~g}$ /day for 3 days in healthy young adults. Clin Pharmacol Ther. 2007;81(6):840-8.

3. Shayiq RM, Roberts DW, Rothstein K, et al. Repeat exposure to incremental doses of acetaminophen provides protection against acetaminophen-induced lethality in mice: an explanation for high acetaminophen dosage in humans without hepatic injury. Hepatology (Baltimore, Md.) 1999;29(2):451-63.

4. Okey AB, Boutros PC, Harper PA. Polymorphisms of human nuclear receptors that control expression of drug-metabolizing enzymes. Pharmacogenet Genomics. 2005;15(6):371-9.

5. Hussaini SA, Farrington EA. Idiosyncratic drug-induced liver injury: an overview. Expert Opin Drug Saf. 2007;6(6):673-84.

6. Bon Homme M, Channareddy S, Tucker W, et al. Repeated exposure to acetaminophen protects against a subsequent lethal dose through selective depletion of cytosolic glutathione. Hepatology (Baltimore, Md.) 2007;46(4S1):805A.

7. O'Brien PJ, Slaughter MR, Swain A, et al. Repeated acetaminophen dosing in rats: adaptation of hepatic antioxidant system. Hum Exp Toxicol. 2000;19(5):277-83.

8. Gardner CR, Laskin JD, Dambach DM, et al. Reduced hepatotoxicity of acetaminophen in mice lacking inducible nitric oxide synthase: potential role of tumor necrosis factor-alpha and interleukin-10. Toxicol Appl Pharmacol. 2002;184(1):27-36. 
9. James LP, Mayeux PR, Hinson JA. Acetaminophen-induced hepatotoxicity. Drug Metab Dispos. 2003;31(12):1499-506.

10. Hogaboam CM, Bone-Larson CL, Steinhauser ML, et al. Novel CXCR2-dependent liver regenerative qualities of ELR-containing CXC chemokines. FASEB J. 1999;13(12):1565-74.

11. Bone-Larson CL, Simpson KJ, Colletti LM, et al. The role of chemokines in the immunopathology of the liver. Immunol Rev. 2000;177:8-20.

12. James LP, Simpson PM, Farrar HC, et al. Cytokines and toxicity in acetaminophen overdose. J Clin Pharmacol. 2005;45(10):116571 .

13. Mirochnitchenko O, Weisbrot-Lefkowitz M, Reuhl K, et al. Acetaminophen toxicity. Opposite effects of two forms of glutathione peroxidase. J Biol Chem. 1999;274(15):10349-55.

14. Dalhoff K, Laursen H, Bangert K, et al. Autoprotection in acetaminophen intoxication in rats: the role of liver regeneration. Pharmacol Toxicol. 2001;88(3):135-41.

15. Lee SS, Buters JT, Pineau T, et al. Role of CYP2E1 in the hepatotoxicity of acetaminophen. J Biol Chem. 1996;271 (20):12063-7.

16. Stalder D, Haeberli A, Heller M. Evaluation of reproducibility of protein identification results after multidimensional human serum protein separation. Proteomics. 2008;8(3):414-24.

17. Hong YJ, Wang XD, Shen D, et al. Discrimination analysis of mass spectroscopy proteomics for ovarian cancer detection. Acta Pharmacol Sin. 2008;29(10):1240-6. Oct.

18. Xhao X, Deyanova EG, Lubbers LS, et al. Differential mass spectroscpopy of rat plasma reveals proteins that are responsive to 17 beta-estradiol and a selective estrogen receptor modulator PPT. J Proteome Res. 2008;7(10):4373-83.

19. Smalley DM, Ley K. Plasma-derived microparticles for biomarker discovery. Clin Lab. 2008;54(3-4):67-70.

20. Zerefos PG, Vlahou A. Urine sample preparation and protein profiling by two-dimensional electrophoresis and matrix-assisted laser desorption ionization time of flight mass spectroscopy. Method Mol Biol. 2008;428:141-57.

21. Rossing K, Mischak H, Dakna M, et al. Urinary proteomics in diabetes and CKD. J Am Soc Nephrol. 2008;19(7):128-90.

22. Wu DL, Zhang WH, Wang WJ, et al. Proteomic evaluation of urine from renal cell carcinoma using SELDI-TOF-MS and tree analysis pattern. Technol Cancer Res Treat. 2008;7(3):155-60. June.

23. Buhimschi IA, Zhao G, Funai EF, et al. Proteomic profiling of urine identifies fragments of SERPINA1 and albumin as biomarkers of preeclampsia. Am J Obstet Gynecol. 2008;199(5):155. e1-16. Nov.

24. Lee AY, Paweletz CP, Pollack RM, et al. Quantitative analysis of histone deacetylase-1 selective histone modifications by differential mass spectroscopy. J Proteome Res. 2008;in press.

25. Pagliarini DJ, Calvo SE, Chang B, et al. A mitochondrial protein compendium elucidates complex I disease biology. Cell. 2008;134 (1):22-4. Jul 11.

26. Sachs AN, Pisitkun T, Hoffert JD, et al. LC-MS/MS analysis of differential centrifugation fractions from native inner medullary collecting duct of rat. Am J Physiol Renal Physiol. 2008;295: F1799-1806.

27. Zhu Y, Valdes R Jr., Jortani SA. Application of bioaffinity mass spectrometry for analysis of ligands. Ther Drug Monit. 2005;27 (6):694-9.

28. Zhu Y, Valdes R Jr., Simmons CQ, et al. Analysis of ligand binding by bioaffinity mass spectrometry. Clin Chim Acta. 2006;371(1-2):71-8.

29. Korecká L, Jankovicová B, Krenková J, et al. Bioaffinity magnetic reactor for peptide digestion followed by analysis using bottom-up shotgun proteomics strategy. J Sep Sci. 2008;31 (3):507-15. Feb.

30. Sinha A, Srivastava N, Singh S, et al. Identification of differentially displayed proteins in cerebrospinal fluid of Parkinson's disease patients: a proteomic approach. Clin Chim Acta. 2008;400:14-20.

31. Süssmuth SD, Brettschneider J, Ludolph AC, et al. Biochemical markers in CSF of ALS patients. Curr Med Chem. 2008;15 (18): 1788-801.

32. Wiemer JC, Prokudin A. Bioinformatics in proteomics: application, terminology and pitfalls. Pathol Res Pract. 2004;200(2):173-8.

33. Gramolini AO, Peterman SM, Kislinger T. Mass spectrometrybased proteomics: a useful tool for biomarker discovery? Clin Pharmacol Ther. 2008;83(5):758-60.

34. Tabb DL. What's driving false discovery rates? J Proteome Res. 2008;7(1):45-6.

35. Linder MW, Prough RA. Developmental aspects of glucocorticoid regulation of polycyclic aromatic hydrocarbon inducible enzymes. Arch Biochem Biophys. 1993;302:92-103. http://www.ptglab. com/datasheet.asp? catNo=10313-1-AP.

36. Collart MA, Struhl K. CDC39, an essential nuclear protein that negatively regulates transcription and differentially affects the constitutive and inducible HIS3 promoters. EMBO J. 1993;12 (1): $177-86$

37. Collart MA, Struhl K. NOT1 (CDC39), NOT2 (CDC36), NOT3, and NOT4 encode a global-negative regulator of transcription that differentially affects TATA-element utilization. Genes Dev. 1994;8 (5):525-37.

38. Chen J, Rappsilber J, Chiang YC, et al. Purification and characterization of the 1.0 MDa CCR4-NOT complex identifies two novel components of the complex. J Mol Biol. 2001;314 (4):683-94

39. Denis CL, Chen J. The CCR4-NOT complex plays diverse roles in mRNA metabolism. Prog Nucleic Acid Res. 2003;73:221-50.

40. Winkler GS, Mulder KW, Bardwell VJ, et al. Human Ccr4-Not complex is a ligand-dependent repressor of nuclear receptormediated transcription. EMBO J. 2006;25(13):3089-99.

41. Jayne S, Zwartjes CG, van Schaik FM, et al. Involvement of the SMRT/NCoR-HDAC3 complex in transcriptional repression by the CNOT2 subunit of the human Ccr4-Not complex. Biochem J. 2006;398(3):461-7.

42. Mazer M, Perrone J. Acetaminophen-induced nephrotoxicity: pathophysiology, clinical manifestations, and management. J Med Toxicol. 2008;4(1):2-6.

43. Stern ST, Bruno MK, Horton RA, et al. Contribution of acetaminophen-cysteine to acetaminophen nephrotoxicity II. Possible involvement of the gamma-glutamyl cycle. Toxicol Appl Pharmacol. 2005;202(2):160-71.

44. Stern ST, Bruno MK, Hennig GE, et al. Contribution of acetaminophen-cysteine to acetaminophen nephrotoxicity in CD1 mice: I. Enhancement of acetaminophen nephrotoxicity by acetaminophen-cysteine. Toxicol Appl Pharmacol. 2005;202 (2):151-9.

45. Ramaiah SK, Jaeschke H. Role of neutrophils in the pathogenesis of acute inflammatory liver injury. Toxicol Pathol. 2007;35 (6):757-66

46. Numata K, Kubo M, Watanabe $\mathrm{H}$, et al. Overexpression of suppressor of cytokine signaling-3 in $\mathrm{T}$ cells exacerbates acetaminophen-induced hepatotoxicity. J Immunol. 2007;178 (6):3777-85.

47. Bourdi M, Eiras DP, Holt MP, et al. Role of IL-6 in an IL-10 and IL-4 double knockout mouse model uniquely susceptible to acetaminophen-induced liver injury. Chem Res Toxicol. 2007;20 (2):208-16. 